\title{
BGleiG und BGremBG - Ergebnisse und Einschätzungen
}

\section{Eva M. Welskop-Deffaa}

Abteilungsleiterin Gleichstellung, Chancengleichheit, Bundesministerium für Familie, Senioren, Frauen und Jugend, Berlin

Es ist mir eine große Freude, heute zum Abschluss Ihrer Fachtagung zu Ihnen zu sprechen. Ihre Veranstaltung zum Thema „Gläserne Decke: Gleichberechtigung im öffentlichen Dienst Anspruch und Wirklichkeit" kommt zum richtigen Zeitpunkt, denn im Rahmen des Stufenplans „Mehr Frauen in Führungspositionen “ arbeiten wir gerade mit Volldampf daran, die Empfehlungen der Berichte zum Bundesgleichstellungsgesetz (BGleiG) und zum Bundesgremienbesetzungsgesetz (BGremBG) aus dem vergangenen Dezember umzusetzen und die gesetzliche Grundlage für Gleichstellung im öffentlichen Dienst - das Bundesgleichstellungsgesetz und das Bundesgremienbesetzungsgesetz - zu überarbeiten.

Sie haben heute im Verlauf des Tages in einer Reihe von Vorträgen und Diskussionsbeiträgen die Situation von Frauen und Männern im öffentlichen Dienst ausführlich beleuchtet und die Faktenlage gründlich dargestellt. Ich möchte daher an dieser Stelle auf eine Wiederholung der Zahlen verzichten und mich bei der Darstellung der Wirklichkeit schlaglichtartig auf drei Besonderheiten des öffentlichen Dienstes beschränken, bevor ich dem Titel Ihrer Tagung folgend - einige Aspekte anspreche, die deutlich machen, an welchem Anspruch sich Geschlechtergerechtigkeit im öffentlichen Dienst heute messen lassen muss.

\section{Die „Gläserne Decke“ in der Bundesverwaltung}

Der Begriff der gläsernen Decke wird sowohl für die Privatwirtschaft als auch für den öffentlichen Dienst verwendet: Der Begriff steht ganz allgemein für die eingeschränkten Aufstiegschancen von Frauen. In der Bundesverwaltung scheint es mir allerdings passender, von gläsernen Kästen zu sprechen. Über 50 Prozent der Beschäftigten in der Bundesverwaltung sind weiblich, knapp ein Drittel der Referatsleitungen ist in Frauenhand und auch auf Unterabteilungsebene sind gewisse Fortschritte zu verzeichnen. Wir sehen Veränderungen, wenn auch langsame. Die Frauen kommen in mittlere Führungspositionen, nicht zuletzt unterstützt durch die Instrumente des BGleiG. Auch an der Spitze der Ministerien, auf Ministerebene, hat sich in den letzten Jahren viel getan. Es ist durchaus beachtlich, dass neben der Kanzlerin auch die Ressortchefs wichtiger Häuser weiblich sind und unter den Kabinettsmitgliedern ein Drittel Frauen sind. Anders allerdings sieht es in den Spitzen der Ministerialbürokratie aus. Hier sind - eingekastelt in den gläsernen Kasten - auf Staatssekretärs- und Abteilungsleiterebene die Frauen eine wirkliche Rarität. Im EU-Vergleich nimmt Deutschland hier eine der Schlusslicht-Positionen ein. Als ich vor fünfeinhalb Jahren Abteilungsleiterin wurde, gab es keine einzige beamtete Staatssekretärin in der Bundesregierung - und so war es seit dem 2. Weltkrieg meist gewesen. Es ist beachtlich, dass die Zahl der beamteten Staatssekretärinnen in dieser Legislaturperiode auf vier gestiegen ist und es spricht einiges dafür, davon auszugehen, dass es in Kürze fünf sein könnten. Ihnen stehen allerdings 25 Männer als beamtete Staatssekretäre gegenüber. Der gläserne Kasten war lange Zeit aus Panzerglas, denn für die politische Leitungsebene im Ministerium gelten besondere Regeln. Hier greifen weder BGleiG und BGremBG, noch funktioniert der politische Druck, der sich in Wahlen äußert und der im Bereich der Abgeordneten und Minister eine gewisse Frauenrepräsentanz seit den Achtzigerjahren verlässlich einfordert. Für die Staatssekretärs- und Abteilungsleitungspositionen sind neben Fähigkeiten, die bei jeder Führungsposition zwingend notwendig sind - Leistungsfähigkeit und Leistungsbereitschaft - politische Netzwerke von großer Bedeutung. Frauen brauchen, um in diese obersten Führungspositionen in der Bundesverwaltung zu gelangen, nicht nur die Bereitschaft zu hervorragenden Leistungen, sondern auch das Engagement in politischen Netzwerken. Nur so wird es gelingen, den gläsernen Kasten zu sprengen.

\section{Heterogenität in der Bundesverwaltung}

Wenn wir, wie im zweiten Erfahrungsbericht, eine Bestandsaufnahme zur Gleichstellung von Frauen und Männern in der Bundesverwaltung vornehmen, fällt auf, dass die Bundesverwaltung sehr heterogen ist. Es gibt Bereiche, da sind die Ziele des BGleiG weit umgesetzt und es gibt Bereiche, da sind wir weit entfernt. In vielen Bereichen ist das Frauenministerium gefragt, in anderen Bereichen - wie in den Gerichten, in der Wissenschaft oder Unternehmen der öffentlichen Hand - haben wir kaum Einfluss. Um gleichberechtigte Teilhabe an Führungspositionen der öffentlichen Hand generell zu fördern, ist es wichtig, die Rolle des Bundesgleichstellungsministeriums als koordinierendem Ressort zu stärken. Unser Vorschlag eines Bundesgleichstellungsplanarchivs zum Beispiel, in dem alle Gleichstellungspläne gesammelt und damit der Prüfung zugänglich gemacht würden, ob sie den Vorgaben des BGleiG tatsächlich entsprechen, ging in eine solche Richtung.

\section{Mangelnde Durchlässigkeit}

Ich möchte Sie noch auf einen Fakt aufmerksam machen, der in der Diskussion um Gleichstellung von Frauen und Männern in der Bundesverwaltung bisher wenig Beachtung findet: die mangelnde Durchlässigkeit zwischen Verwaltung und Privatwirtschaft und die mangelnde Durchlässigkeit zwischen den Ressorts. Es gibt - anders als in Nachbarländern - kaum Wechsel zwischen den Ressorts und praktisch keine Wechsel von der Verwaltung in die Wirtschaft (und zurück) - Karriere wird meist in einem Ministerium gemacht. Das sind sogenannte Kaminkarrieren. In der Privatwirtschaft ist ein bewährtes Mittel zur Karrierebeschleunigung der Unternehmenswechsel. Und das gilt auch für Frauen. Im öffentlichen Dienst gibt es solche Wechsel kaum. Das sollte sich in Zukunft ändern. Zudem sollten Wechsel zwischen öffentlichem Dienst und Privatwirtschaft normaler werden. Frauen sollten sich nicht in den öffentlichen Dienst zurückzie- 
hen. Wir sehen an anderen europäischen Ländern wie beispielsweise Dänemark, dass eine solche Segregation zwar bequem sein kann, aber langfristig Frauen in eine Sackgasse schiebt.

Soweit zur Wirklichkeit. Und nun zum Anspruch, an dem sich Gleichberechtigung und Gleichstellungspolitik (für die Bundesverwaltung ebenso wie für die Privatwirtschaft) in Deutschland heute messen lassen muss. Es fällt uns nicht ganz leicht, den Maßstab zu benennen, an dem sich der Anspruch unbestritten festmachen ließe. Anders als in Frankreich: Dort besteht eine große Einmütigkeit, dass der Maßstab, an dem sich Gleichberechtigung messen lassen muss, „parité“ ist - die paritätische Besetzung von Positionen. Gleichberechtigung bedeutet in Frankreich, dass alle Positionen und Ämter in der Gesellschaft, in der Politik und in der Wirtschaft zu gleichen Teilen von Frauen und Männern ausgeübt werden sollten. In Deutschland sehen wir die Sache nicht so schlicht. Gleichberechtigung wird nicht einfach mit Parität gleichgesetzt.

Der schlichte Statusvergleich, die einfache zahlenmäßige Gleichbehandlung von Frauen und Männern kommt ein wenig (zu) simpel daher. Unsere Anstrengungen in einer modernen Gleichstellungspolitik gehen stattdessen - wie es auch der Erste Gleichstellungsbericht der Bundesregierung entwickelt - dahin, Chancen und Risiken im Lebensverlauf in den Blick zu nehmen. Weichenstellende Übergänge sind so zu gestalten, dass sie keine Narbeneffekte hinterlassen und geschlechtshierarchisch verteilte Nachteile mit sich bringen. Die Gleichstellungsberichtskommission spricht vom Konzept der „linked lives“. Wir müssen sehen, dass viele Entscheidungen, die das Erwerbsleben und die Lebensperspektiven von Frauen grundsätzlich beeinflussen, in Partnerschaften - von Frauen und Männern gemeinsam - getroffen werden. Frauen nehmen oftmals bei ihren Entscheidungen über Teilzeit-, Vollzeitbeschäftigung oder Karrierechancen Rücksicht auf die Karriereambitionen des Partners. Zu oft schwingt bei solchen Entscheidungen das Bild der Partnerin mit, die dem Mann den Rücken freihält. Dabei werden kurzfristige Auswirkungen von Entscheidungen oftmals höher gewichtet als die langfristigen. Kurzfristig kann eine Teilzeitbeschäftigung die Vereinbarkeit von Erwerbsleben und Beruf verbessern, langfristig bedeutet sie einen Einschnitt in der weiteren Karriereentwicklung und eine irreversible Verschlechterung der Alterssicherung. Eine moderne Gleichstellungspolitik muss dem Anspruch genügen, den gesamten Lebensverlauf von Frauen und Männern zu berücksichtigen.

Abschließend möchte ich kurz noch auf die Instrumente zu sprechen kommen, die dazu angetan sein könnten, Anspruch und Wirklichkeit zu versöhnen. Der Gesetzgeber hat im Bundesgleichstellungsgesetz mit der Verpflichtung zur Vorlage von Gleichstellungsplänen ein grundsätzlich wirkungsvolles Instrument geschaffen. Leider wird das Potenzial des Gleichstellungsplans als Steuerungsinstrument von der Personalverwaltung keineswegs durchgängig richtig erkannt. In den meisten dieser Pläne wird in epischer Breite die Ist-Situation von weiblichen und männlichen Beschäftigten dargestellt - Statistiken zum Frauenanteil in allen möglichen Bereichen der Verwaltung. Dafür haben sich die Personalabteilungen, die maßgeblich an der Erarbeitung mitwirken, Fleißpunkte verdient. Aber damit ist den gesetzlichen Vorschriften in keinem Fall genüge getan. Gleichstellungspläne sollten ein Planungsinstrument sein - also vor allem in die Zukunft gerichtet sein. Die zentralen Bestandteile sollten konkrete Zielvorgaben und Maßnahmen zur Erreichung dieser Ziele sein. Leider sind Gleichstellungspläne, die verbindliche Zielvorgaben und konkrete Maßnahmen enthalten, wie eine Stecknadel im Heuhaufen - man findet sie nicht.

In Zukunft wollen wir die Erarbeitung der Gleichstellungspläne aktiv durch einen Mustergleichstellungsplan und einen Leitfaden unterstützen. Außerdem werden wir die Gleichstellungspläne mit einem jährlichen Monitoring eng verzahnen. Dieses Monitoring, das wichtige Kennzahlen zur Gleichstellung, zur Vereinbarkeit und zur Gremienbesetzung enthalten soll, ermöglicht es, die jährlichen Entwicklungen nachzuvollziehen und Vergleiche zwischen den Ressorts vorzunehmen.

Die Gleichstellungsbeauftragte ist eine der wichtigsten Akteurinnen für die Gleichstellung im öffentlichen Dienst. Das Bundesgleichstellungsgesetz sieht die Wahl einer Gleichstellungsbeauftragten vor. An der Wahl werden wir festhalten, weil sie durch eine Wahl als Vertreterin der Mitarbeiter für Gleichstellung legitimiert wird und in der Belegschaft verankert ist. Allerdings haben wir vor, das Amt der Gleichstellungsbeauftragten auch für Männer zu öffnen und männliche Mitarbeiter an der Wahl zu beteiligen, sofern die weiblichen Mitarbeiterinnen einer Dienststelle dies befürworten. Dahinter steckt der Gedanke, dass diese Öffnung zu einer breiten Akzeptanz der Gleichstellungsbeauftragten oder des Gleichstellungsbeauftragten führt. Gleichstellung ist kein Frauenthema, sondern geht Frauen und Männer gleichermaßen an. Im Übrigen gab es bereits eine Phase, zu Zeiten des Frauenfördergesetzes, in der auch Männer dieses Amt bekleiden durften. Es gibt keine Hinweise, dass Männer als Gleichstellungsbeauftragte das Ziel der Gleichstellung weniger effektiv verfolgt hätten.

Die Gleichstellungsbeauftragte ist natürlich nicht die einzige Akteurin. Neben ihr sind die Personalverwaltung und vor allem die Führungskräfte verpflichtet, die Gleichstellung von Frauen und Männern zu fördern. Leider ist die Zusammenarbeit dieser Akteurinnen und Akteure noch ausbaufähig. Es herrschen große Rollenunsicherheiten, zu deren Klärung wir in der nächsten Zeit beitragen wollen.

Das Reden ist die eine Sache. Wenn dem keine Taten folgen, dann hinterlassen Worte nur einen schalen Nachgeschmack. Wir sind willens, die Gleichstellung einen großen Schritt voranzubringen. Dazu benötigen wir Unterstützung. Wir brauchen mutige und kreative Partner wie den djb. Unser gemeinsames Projekt „Aktionärinnen fordern Gleichberechtigung “ ist in meinen Augen ein sehr gutes Beispiel für eine erfolgreiche $\mathrm{Zu}$ sammenarbeit. Die Idee war ungewöhnlich, aber zündend. Das Medienecho ist groß und hat uns geholfen, das Thema „Frauen in Führungspositionen “ mit Nachdruck voranzutreiben. Ich freue mich auf die Fortsetzung des Projektes, das wir gerne fördern, und hoffe, dass von der heutigen Tagung auch für die Förderung von Frauen im öffentlichen Dienst neue Impulse ausgehen. Vielen Dank. 